\title{
OPACITY SOURCES IN THE UV SPECTRUM \\ OF THE SUN
}

\author{
R. M. BONNET and D. SACOTTE \\ CNRS Laboratoire de Physique Stellaire et Planétaire, Verrières-le-Buisson, France
}

\section{Introduction}

In the past three or four years a great deal of attention has been given to the interpretation of the solar UV continuum. Several sets of intensity and limb-darkening measurements made from satellite, rocket and balloon borne instruments are now available, which allow comparisons to be made with theoretical computations. The main purpose of this paper is to discuss these comparisons, we restrict this discussion to the wavelength region between $3000 \AA$ and $1680 \AA$, in which a significant difference between the observations and the computations still exists. The lines in this wavelength region which are of relevance to the continuum opacity are listed as follows:

the Ly- $\alpha$ line of neutral hydrogen at $1216 \AA$ which appears as a strong emission line;

the resonance doublet of $\mathrm{Mg}$ II at $2795 \AA$ and $2803 \AA$ which causes a broad depression in the solar continuum and shows an emission core similar in shape to the $\mathrm{Ly}-\alpha$ line;

the auto-ionization doublet of AlI at $1932 \AA$ and $1936 \AA$, which appears as two broad absorption features;

several absorption lines such as Mg I $2852 \AA$ Si $2881 \AA$;

many FeI and FeII absorption lines.

There are also several discontinuities in the continuum emitted between the lines in the spectrum between $1216 \AA$ and $3000 \AA$. The most important ones are located at $2500 \AA, 2085 \AA$ and $1680 \AA$, which correspond to the photoionization edges of MgI, AlI and SiI respectively.

\section{The Comparison of Computed and Observed Spectra}

In order to compute the spectrum one must make use of a model atmosphere which usually describes the variation with altitude in the atmosphere of the electron temperature, the electron pressure, the total pressure, the chemical composition and stage of ionization of each element. The emerging intensity, $I_{\lambda}$, at the center of the solar disk at wavelength $\lambda$ is given by the integral:

$$
I_{\lambda}=\int_{0}^{\infty} S_{\lambda}\left(\tau_{\lambda}\right) e^{-\tau_{\lambda}} \mathrm{d} \tau_{\lambda} .
$$


If Local Thermodynamic Equilibrium (LTE) can be assumed we have the very simple identity: $S\left(\tau_{\lambda}\right) \equiv B_{\lambda}(T)$. The parameter $\tau_{\lambda}$ represents the optical depth at wavelength $\lambda, T$ is the temperature.

The optical depth, $\tau_{\lambda}$, is also the result of an integration over the geometrical depth $z$ of the absorption coefficient, $\chi_{\lambda}$, at wavelength $\lambda$.

If one wants to compute the continuous spectrum only, the value of $\chi_{\lambda}$ is the addition of all the absorption coefficients of the elements which might contribute to the opacity at wavelength $\lambda$. These coefficients can be either computed theoretically by means of quantum mechanics or can be measured in the laboratory. Sometimes, only the value at the threshold is available. In this case, an assumption must be made as to how the coefficient varies with wavelength. If discrepancies appear between the result of the computations and the observations one must question the accuracy of model used (including the abundances used in the chemical composition), the validity of the assumption of LTE and the accuracy of absorption coefficient. If none of these quantities is certain then the problem remains entirely undetermined at the wavelength considered. This is the case for the spectral range which is considered here. Therefore, an iterative procedure must be undertaken.

Fortunately, the layers from which the UV radiation is emitted also radiate in the infra-red between about $10 \mu$ and $300 \mu$ where, except for a few regions, the absorption coefficient is perfectly well known and the LTE assumption can be shown to be valid. Therefore the models can be tested in the IR, and the absorption coefficient in the UV can be determined assuming that LTE also holds in this region.

In a previous paper (Bonnet, 1968) we compared the empirical values of the solar opacity, deduced from limb-darkening measurements in the ultra-violet between $2000 \AA$ and $3000 \AA$, with theoretical values, computed using the Bilderberg Continuum Atmosphere (Gingerich, 1968). We showed that a substantial amount of extra opacity is present in the continuous spectrum of the Sun below $3500 \AA$. As a result of this underestimation of the continuous opacity the computed values of the emerging intensities are systematically higher than the measured values. Until recently three major discrepancies could be seen between the observations and the computations of the ultra-violet intensity of the Sun.

(a) The Ali photoionization discontinuity could not allow for the strong drop in intensity at $2085 \AA$ observed in the solar spectrum.

(b) The Sil photoionization discontinuity at $1680 \AA$ shows the opposite situation and the observed intensity drop is not so strong as that predicted.

(c) The observed values of the solar intensity lie systematically lower than the predicted values between $3500 \AA$ and $1680 \AA$.

These three points will be discussed in turn.

\section{The AlI Discontinuity}

The AlI discontinuity has been measured recently from a high resolution spectrum obtained at Culham (Boland et al., 1970). The intensity drops by a factor of 5 between 
$2100 \AA$ and $2080 \AA$, in good agreement with previous determinations ranging between 5 and 6 (Bonnet, 1968). The computed value of the drop using the BCA model is a factor of 2 smaller. The difference between the observed position in wavelength of the discontinuity, close to $2085 \AA$, and the theoretical position of the photoionization limit of $\mathrm{Al}$ I at $2071 \AA$, is also apparent. Therefore the question arises as to whether or not $\mathrm{Al} \mathrm{I}$ is the element whose photoionization from the ground state is responsible for the observed drop in the solar UV continuum. Boland et al. have shown theoretically that the intensity of the Alı photoionization edge is a very sensitive function of the temperature of the layers of the atmospheres where it occurs. Their conclusion is that a decrease in the temperature of the Bilderberg model is sufficient to represent both the observed intensity decrease and the shift in the position of the All absorption edge. This conclusion is in good agreement with the observations made in the IR by Lena (1969), which need a model with a temperature minimum close to $4300 \mathrm{~K}$. Such a model also agrees with the photoelectric measurements of the solar intensity below $1800 \AA$ made by Parkinson and Reeves (1969). However, the limb-darkening variation of the discontinuity has not been discussed, and the appearance of the chromospheric network just below the absorption edge has also to be discussed further before the discontinuity can be assigned definitely to the photoionization of aluminium.

\section{The SiI $\left(3 p^{2}{ }^{1} D\right)$ Photoionization Edge at $1680 \AA$}

The situation at $1680 \AA$ is quite different from that at $2080 \AA$. The Sil photoionization edge at $1680 \AA$ is predicted to give an intensity drop of more than a factor of ten. Unfortunately this does not agree at all with the observations. However, the observed spectrum changes radically at this wavelength; limb-darkening vanishes on the blue side of the limit and also absorption lines are virtually absent. Both characteristics are well predicted by models. As a consequence of the presence of emission lines, the level of the continuum is difficult to locate and the intensity of the Sir drop cannot easily be evaluated. It is important to notice that there exists a similar situation in the spectrum of Sirius, as revealed in the spectra obtained with the Orbiting Astronomical Observatory (Code, 1970), where the discontinuity can barely be detected, whereas computations predict a large drop.

The value of 35 megabars for the absorption cross-section at the edge as measured by Rich (1966) seems to be reliable. The discussion by Boland et al. on the AlI discontinuity shows that the intensity of the Sil drop would not be sensitive to a change in temperature. Therefore, the temperature distribution of the model cannot be made responsible for the disagreement. Furthermore, decreasing the temperature of the model in this region would lead to serious disagreements in the IR region.

If it is assumed that the opacity has been underestimated in the region above $1800 \AA$, the fact that all the observed points lie below the predicted values can be explained.

Therefore, the following question arises: 


\section{Is There an Unknown Source of Continuous Opacity in the Near UV?}

From the above discussion one is tempted to say, yes!

\section{A. THE WINGS OF THE LY- $\alpha$ LINE OF H I}

In 1968 Cuny (1968) reported, at the third Harvard Conference on Stellar Atmospheres, that the resonance broadening of the $L y-\alpha$ line of hydrogen would cause the wings of this line to be an important source of opacity even at $3000 \AA$ from the centre of the line. Cuny adopted the conventional formula for the resonance contribution in order to compute the absorption coefficient in the wings of the line. As a result, there was perfectly good agreement below $1680 \AA$ and it was shown that the discrepancy could be substantially removed by the inclusion of this source of opacity in the absorption coefficient above this wavelength.

Unfortunately Sando et al. (1969) have shown that the use of the conventional formula seriously overestimates the magnitude of the absorption at long wavelengths. The quasi-static description which leads to the conventional formula is valid in the wings of the line, but the assumption that the interaction varies as the inverse cube of the distance is correct only at long internuclear distances. The above authors show that the use of the conventional formula fails at wavelengths longer than $1228 \AA$ and that no contribution to the opacity can be expected above $1623 \AA$. Therefore a new source of extra opacity has to be found above this wavelength. Carbon monoxide and iron bound-free absorption have been suggested as good candidates (Gingerich, 1969), but no quantitative evaluation is available. Linsky (1970) has shown that the $\mathrm{H}_{3}^{+}$ion could also absorb in this region; however, quantitative evaluations indicate that the contribution of this ion is too small.

\section{B. THE LINE-BLOCKING}

It has often been suggested that the lines in the part of the solar spectrum under consideration here are so numerous that it is practically impossible to observe the continuum. This remark is valid for absorption lines as well as for emission lines. If the concentration of lines throughout the spectrum is really overwhelming, it is easy to understand why the Sil edge at $1600 \AA$ is so weak; at wavelengths longer than the SiI edge the blanketing tends to lower the continuum level; conversely the apparent continuum level is raised on the blue side of the edge because most of the lines are in emission. If this is the only explanation, one might infer from it that the abnormally low intensity of the SiI drop in the spectrum of a star where it is predicted to be strong indicates the presence of a chromosphere in the atmosphere of this star.

We have attempted to estimate the effect of line-blocking on the level reached by the continuum in the range $2000 \AA-3000 \AA$, where a substantial number of oscillator strengths values for the lines are available. We have included in the expression of the absorption coefficient the contribution of the lines computed classically by means of the Hjerting function (Aller, 1963). We have assumed LTE and considered that the temperature distribution of the model would not be modified by the blanketing, since 
this region of the spectrum contributes only a few percent to the total radiative energy output. However, we agree that these two assumptions are crude and subject to many criticisms. The details of this computation have been reported in Bonnet (1968). More than 2400 lines were included in the programme, of which fifty percent are due to FeI and FeII, 800 lines are very weak due to their high excitation energies. Most of the oscillator strengths were taken from Corliss and Bozman (1962); however, when available, a correction has been applied to these values following Warner (1967), who estimates that most of the Corliss and Bozman oscillator strengths are overestimated, sometimes by as much as a factor of 10 .

Recently (Sacotte 1970), we have improved this computation by convoluting the computed spectra with the instrumental profile of our observed spectra (Bonnet et al.,

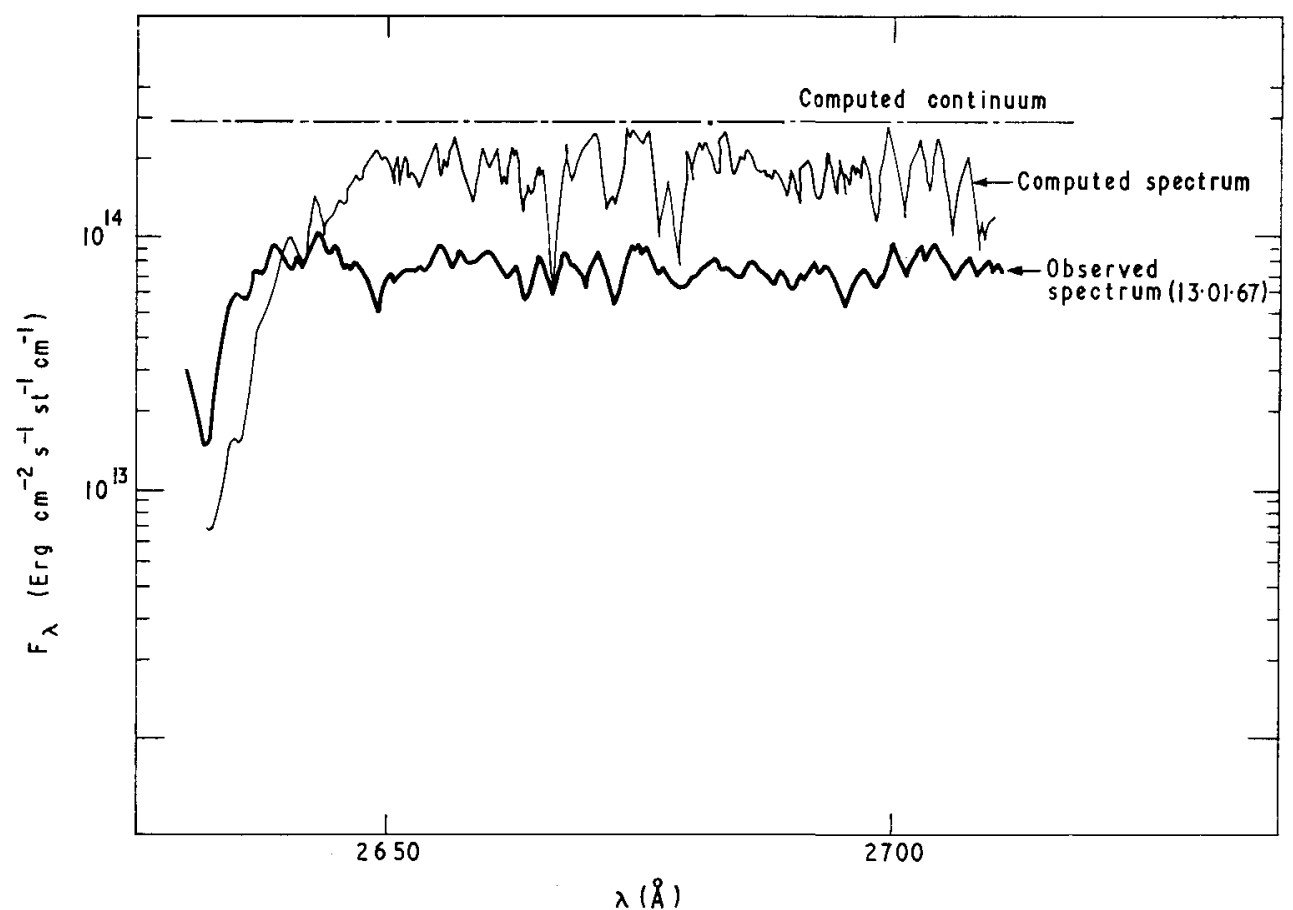

Fig. 1. Comparison between the observed spectrum of January 13,1967 and the computed spectrum. The computed spectrum has been convoluted with an instrumental profile. The resolution of both spectra is $0.4 \AA$. The $g f$-values of Corliss and Bozman have been used here.

1967) with which the results are compared. The BCA model was used. The result of the computation is shown in Figure 1, where the disagreement with the observations is obvious. In Figure 1 the original $f$-values of Corliss and Bozman have been used in order to have an upper limit on the effect of line-blocking. Not only are the observed points lower than the computed ones but the detailed shape of the upper envelope of the observed points is not correctly represented. We have shown that 
the most important contribution to line blocking is due to the wings of strong lines and as a result we show that the theoretical continuum is never reached in the region between $2630 \AA$ and $2750 \AA$ where we have made our more detailed computations. We then define what we call a pseudo-continuum which is the envelope of the higher points. We also show that the introduction of the weak lines in the computation affects this pseudo-continuum locally, but has no influence on its overall shape. However, this conclusion may not be valid if the number of weak lines is increased. In order to check the consistency of the computation we have included into the programme the value of the continuous absorption that we have determined empirically. The result is shown in Figure 2. We see that the observed and computed intensities reach the same level and that some characteristic details are well represented; for example, this is the case for the two emission peaks at $2650 \AA$. Therefore, the inclusion of this extra opacity improves the agreement with the observations.

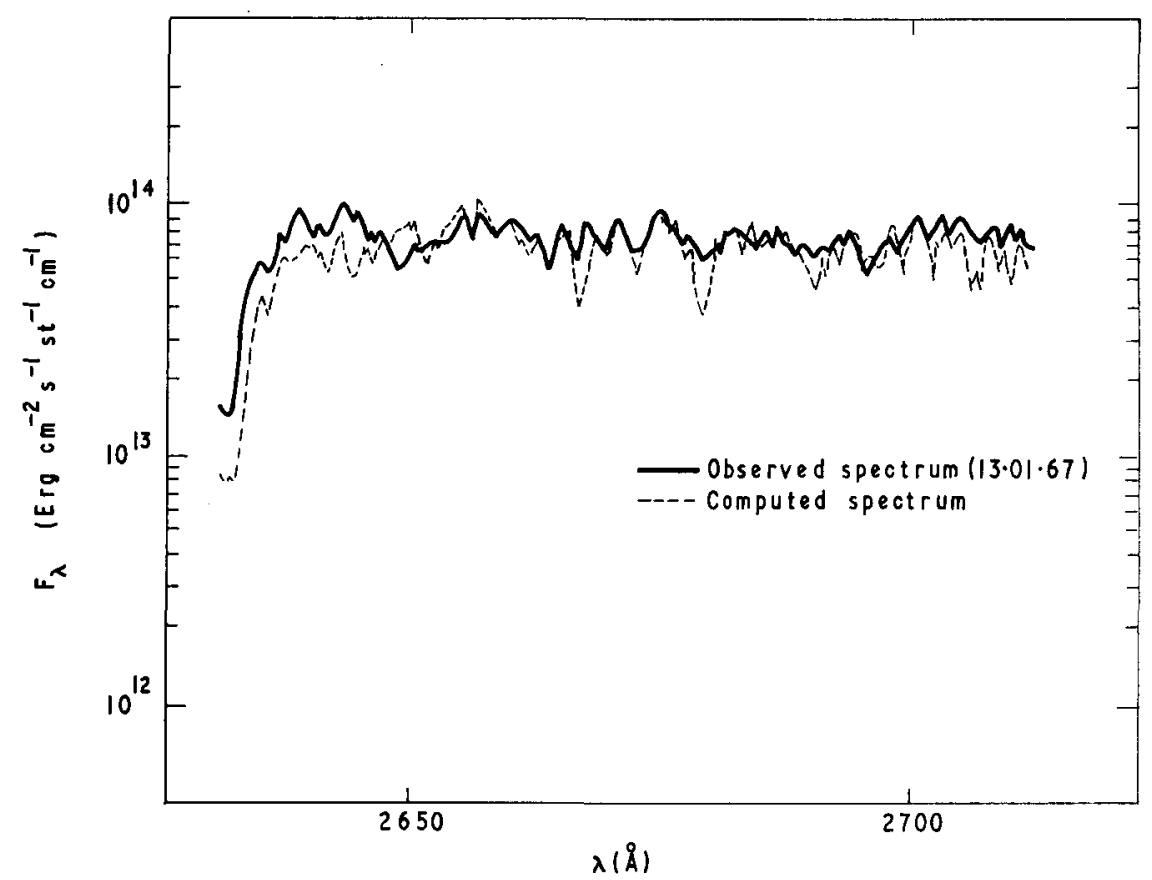

Fig. 2. Same comparison as in Figure 1. The extra opacity determined from limb-darkening measurements has been added to the other known opacity sources in the computation.

Are we able to draw a conclusion at this point on the line-blocking effect? As shown in Figure 3, we have found that the extra opacity determined from limb darkening measurements is composed of;

(a) a component which varies linearly with wavelength,

(b) a broad and symmetrical feature with a peak at $2500 \AA$.

Because the number of lines increases nearly linearly as we go further in the ultra- 
violet they could contribute to the first component of the extra opacity but it is hard to believe that blanketing could lower the continuum by a factor of 3 .

Is the feature at $2500 \AA$ due to a broad blend of lines? The spectrum of Sirius shows the same depression around this wavelength. Of course there is a large number of FeII lines in this region but why would they lead to such a symmetrical feature? Is this due to the photo-ionization cross-section of $\mathrm{Mg}$ I whose shape has been too crudely simplified in the programme or is it something different?

A careful analysis of this feature, in particular, how it varies with temperature, might cast some light on the presence of an extra opacity source. This analysis is now in progress.

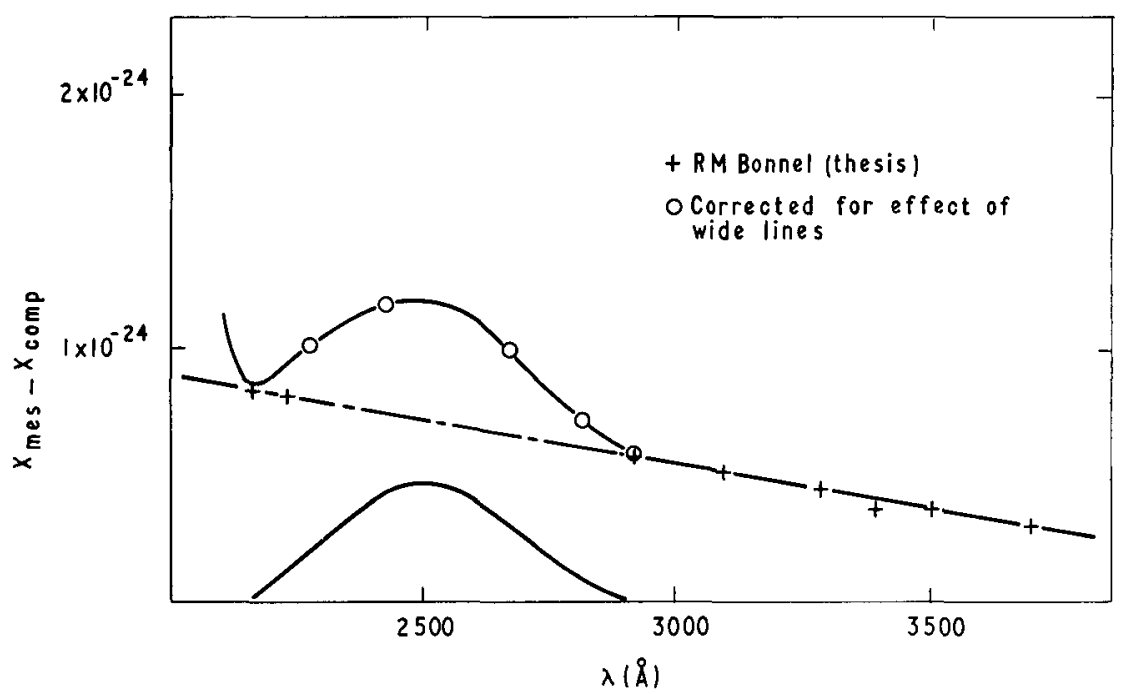

Fig. 3. Variation with wavelength of the extra opacity determined from limb-darkening measurements. The lower curve represents the difference between the extra opacity and the dashed straight line. The temperature used was $5950 \mathrm{~K}$.

\section{Conclusion}

What is needed to correctly reproduce the ultra-violet spectrum of the Sun between $3500 \AA$ and $1680 \AA$ ? First, astrophysicists must provide:

(a) a model atmosphere which has to be checked very carefully in the infra-red,

(b) a theory for computing the intensity of the lines without the assumption of LTE.

Secondly, the spectroscopists must increase the accuracy of the oscillator strengths and provide more of them, and must do more work on the photoionization crosssections at the edges and on both sides of the edges. The observers can also contribute by obtaining high resolution and well calibrated spectra, not only of the Sun but also of the stars, specially in the region around $2500 \AA$. An evaluation of blocking by weak lines has to be made. We think that this can only be done by statistical methods. 
Finally the emerging intensity can be computed and compared with the observations specially at those points where it seems that the continuum is reached.

\section{References}

Aller, L. H.: 1963, Atmospheres of the Sun and Stars (2nd edition), Ronald Press.

Boland, B. C., Jones, B. B., Wilson, R., Engstrom, S. F. T., and Noci, G.: 1971, Phil. Trans. Roy. Soc. A. 270, 29.

Bonnet, R. M.: 1968, Ann. Astrophys. 31, 597.

Bonnet, R. M., Blamont, J. E., and Gildward, P.: 1967, Astrophys. J. 148, L115.

Code, A. D.: 1970, Communication to IAU Symposium 41, Munich.

Corliss, C. H. and Bozman, W. R.: 1962, Nat. Bur. Std. Monograph No. 53.

Cuny, Y.: 1968, Proceedings of the Third Harvard Smithsonian Conference on Stellar Atmosphere, 173.

Gingerich, O. and de Jager, C.: 1968, Solar Phys. 3, 5.

Gingerich, O.: 1969, Bull. Ann. Astron. Soc. 1, 227.

Lena, P.: 1969, Astron. Astrophys. 4, 202.

Lena, P.: 1970, Solar Phys. 10, 330.

Linsky, J. L.: 1970, Solar Phys. 11, 198.

Parkinson, W. H. and Reeves, E. M.: 1969, Solar Phys. 10, 342.

Rich, J. C.: 1966, Thesis, Harvard University, Cambridge, Mass.

Sacotte, D. and Bonnet, R. M.: to be published.

Sando, K., Doyle, R. O., and Dalgarno, A.: 1969, Astrophys. J. 157, L143.

Warner, B.: 1967, Mem. Roy. Astron. Soc. 70, 165. 